\title{
The Nature of Dark Energy and Dark Matter
}

\author{
Ranku Kalita
}

6A, Gopal Road, Panbazar, Guwahati, 781001, India

\begin{abstract}
It is proposed that the accelerating expansion of the universe is due the cosmologicalization of the principle of equivalence - which recognizes momentary equivalence between gravitational force and inertial force - in an expanding spherical distribution of matter, wherein momentary equivalence occurs at a certain radial distance between gravitational force upon the gravitational mass and inertial force - generated by the accelerating frame of reference of spacetime - upon the inertial mass of an object on the surface of the sphere. The principle of equivalence also recognizes momentary equivalence between a freefalling gravitational mass and the impression of acceleration of an inertial mass in an inertial frame of reference with respect to an accelerating frame of reference. Since the acceleration of the inertial mass is impressional, it is conceivable therefore that the inertial mass of the object can be impressional as well. It is proposed that there are objects in nature which possess impressional inertial mass, and that such objects are the dark matter in the universe.
\end{abstract}

Keywords Cosmology, Dark energy, Dark matter, Cosmological constant

\section{Introduction}

Dark energy, which presently constitutes about $72 \%$ of the universe, is accelerating the expansion of the universe [1-3]. Dark matter, which presently constitutes about $23 \%$ of the universe [3], has so far been only gravitationally detected. While dark energy and dark matter are generally viewed as distinct phenomena, the present proposition considers them as the cosmological manifestations of a single principle - the principle of equivalence.

It is proposed that the antigravitational force that is accelerating the expansion of the universe exists because the principle of equivalence - which recognizes momentary equivalence between gravitational force and inertial force is cosmologicalized in an expanding spherical distribution of matter, whereby spacetime itself is the antigravitational accelerating frame of reference that generates inertial force upon the inertial mass of an object on the surface of the sphere, and which is momentarily equivalent at a certain radial distance to the gravitational force experienced by the gravitational mass of the object.

The principle of equivalence also recognizes momentary equivalence between a freefalling gravitational mass and the impression of acceleration of an inertial mass in an inertial frame of reference with respect to an accelerating frame of reference. Since the acceleration of the inertial mass is impressional, it is conceivable therefore that the inertial mass of the object can be impressional as well. It

* Corresponding author:

rankukalita@gmail.com (Ranku Kalita)

Published online at http://journal.sapub.org/astronomy

Copyright (C) 2014 Scientific \& Academic Publishing. All Rights Reserved is proposed that there are objects in nature which possess impressional inertial mass, and that such objects are the dark matter in the universe.

\section{Dark Energy}

The accelerating expansion of the spacetime universe, parametrized by the cosmological constant $\Lambda$, involves acceleration between objects which increases in proportion to distance between the objects. Because of the low value of $\rho_{\Lambda}$ and because $\rho_{\Lambda} \propto a^{0}$ while $\rho c^{2} \propto a^{-3} \quad\left(\rho_{\Lambda}\right.$ is energy density of $\Lambda$, as in $\rho_{\Lambda}=\Lambda c^{2} / 8 \pi G, a$ is expansion or scale factor, and $\rho c^{2}$ is energy density of matter) the accelerating expansion of the universe becomes evident at late times when $\Omega_{\Lambda}>\frac{1}{2} \Omega_{\mathrm{M}}$ (where $\mathrm{M}$ is matter), which occurred $\sim 6 \times 10^{9}$ years ago.

We have from one of the pair of Friedmann equations:

$$
\frac{\ddot{a}}{a}=-\frac{4 \pi G}{3}\left(\rho+3 P / c^{2}\right)+\frac{\Lambda c^{2}}{3},
$$

which may be re-framed as an equation of motion of an object of mass $m$ on the surface of a sphere of radius $R \equiv a$ and mass $M$

$$
\ddot{R}=-\frac{G M}{R^{2}}+\frac{\Lambda c^{2}}{3} R
$$

[Total 'gravitating mass' $\left.M=\frac{4 \pi}{3} R^{3}\left(\rho+3 P / c^{2}\right)\right]$

The equation of motion determines the gravitational acceleration and antigravitational acceleration on a mass $m$ 
on the surface of a sphere. The antigravitational acceleration is due to $\Lambda c^{2} / 3 R$.

In an expanding spherical distribution of matter $M$, at a certain radial distance, momentary equilibrium occurs between the gravitational force experienced by the gravitational mass of the object $m$, and the antigravitational force experienced by the object $m$. Now if we were to consider the antigravitational acceleration as involving the inertial mass of object $m$, then the antigravitational acceleration would generate inertial force upon the inertial mass of object $m$, and which would be directed toward the centre of the sphere - and which would be momentarily equivalent at a certain radial distance of the expanding sphere with the gravitational force experienced by the gravitational mass of the object $m$. It is proposed that this constitutes the cosmologicalization of the principle of equivalence, which recognizes momentary equivalence between: 1. (a) The gravitational force experienced by the gravitational mass of an object situated on a gravitational surface and (b) the inertial force generated on the inertial mass of an object in a uniformly accelerating frame of reference.

The inertial mass object in 1(b) is in an accelerating frame of reference with respect to the background spacetime, and the acceleration of the object has to be imparted by an independent force. In the cosmologicalization of the principle of equivalence in the expanding spherical distribution of matter, it is spacetime itself that is the accelerating frame of reference of the inertial mass of object $m$, wherein the acceleration is parametrized by $\Lambda c^{2} / 3 R-$ thus obviating the need for an independent force to impart acceleration. In the principle of equivalence the acceleration of the inertial mass object in 1(b) is uniform; in the cosmologicalization, the inertial mass of object $m$ is subject to non-uniform acceleration parametrized by $\Lambda c^{2} / 3 R$.

In the principle of equivalence the momentary equivalence between inertial force on the inertial mass of an object and gravitational force on the gravitational mass of an object is demonstrated by considering the object in two distinct frames of reference - once as a static gravitational mass, in 1(a), and then as a dynamic inertial mass, in 1(b); while in the cosmologicalization, the momentary equivalence between the gravitational and the inertial force on the gravitational and inertial mass of an object $m$ on the surface of an expanding sphere is available at a certain radial distance with the same dynamic object $m$.

The correlation between inertial mass and the cosmological constant can be clearly demonstrated by considering eq. (1) for a static universe.

In a static universe, $\ddot{a}=0$, and assuming a pressureless universe, we have

$$
\frac{\Lambda c^{2}}{3}-\frac{4 \pi G \rho_{m}}{3}=0
$$

Here the negative term $\rho_{m}$ represents gravitational mass, and is counterrelated to the positive antigravitational $\Lambda$ term. Now if the $\rho_{m}$ term is shifted RHS, we have

$$
\Lambda c^{2}=4 \pi G \rho_{m}
$$

Here, the fact that the antigravitational term is correlated to the gravitational term is physically contradictory. This can be resolved, however, by recalling the equivalence of gravitational and inertial mass, whereby we can then consider the $\rho_{m}$ term in terms of inertial mass, which then presents no physical contradiction in its correlation with the $\Lambda$ term in eq. (4).

It is proposed that the inertial mass of an object in the spacetime accelerating frame of reference is manifested in the materiality of the object, and that such objects are the visible matter in the universe.

\section{Dark Matter}

Dark matter continues to elude direct detection. If this situation should persist, however, we would then have to consider if dark matter may in fact be directly undetectable. The following proposes how dark matter may be impossible to detect directly.

Direct detection requires the collision between the inertial mass of visible and dark matter and the scattering of visible matter particles. For direct detection not to occur scattering of inertial mass of visible matter would have to not occur. The question then becomes: Could there be something different between the inertial mass of visible and dark matter?

Visible matter and dark matter both possess gravitational mass. The equivalence of gravitational and inertial mass is manifested in the universality of freefall. The universality of freefall is contained in the weak principle of equivalence. The weak principle of equivalence considers a gravitational mass in freefall. Apart from momentary equivalence between gravitational force and inertial force, the principle of equivalence also recognizes momentary equivalence between: 2(a) A gravitational mass object in freefall and 2(b) a stationary inertial mass object with respect to a uniformly accelerating frame of reference, and whereby the impression of acceleration of the inertial mass object is created.

Since the impression of acceleration of the stationary inertial mass object with respect to an accelerating frame of reference in 2(b) is equivalent to the gravitational mass object in freefall in 2(a), the acceleration of the gravitational mass object can therefore also be considered to be impressional; and, since the freefalling gravitational mass object in 2(a) possesses equivalent inertial mass, the acceleration of the inertial mass of the object can be considered to be impressional as well.

Inertial mass of an object is defined as the resistance of an object to acceleration, as in 1(b). It is not necessary, however, that $1(\mathrm{~b})$ has to be the first principle description of resistance 
of an object to acceleration. We may equally well consider 2(a) or 2(b) as the first principle description of an object that is offering resistance to acceleration, since the principle of equivalence itself recognizes it is sufficient for an object to offer resistance to impressional acceleration in 2(a) or 2(b) for its inertial mass to be described, and for the inertial mass to be equivalent to the gravitational mass of the object. Now this opens up the possibility of considering an object that only offers resistance to impressional acceleration in 2(a) or 2(b). If such an object were to exist, its inertial mass would then have to be described as impressional as well - we may call such an object an impressional inertial mass object.

To recap: An inertial mass object offers resistance to acceleration, as in 1(b), as well as to impressional acceleration, as in 2(a) and 2(b). While the here-proposed impressional inertial mass object only resists impressional acceleration, as in 2(a) and 2(b) - and cannot therefore be considered as an inertial mass object resisting acceleration, as in 1(b).

It is proposed that a freefalling impressional inertial mass object in 2(a) of the principle of equivalence is cosmologicalized in an expanding spherical distribution of matter, whereby an impressional inertial mass object on the surface of the sphere experiences cosmological freefall, whose acceleration is parametrized by $\Lambda c^{2} / 3 R$.

In the principle of equivalence, an inertial mass object is considered in distinct accelerating frames of reference, once in 1(b), and then in freefall in 2(a). In the cosmologicalization, because the spacetime accelerating frame of reference is the same for both an inertial mass object and the here-proposed impressional inertial mass object, it is only an impressional inertial mass object that is in cosmological freefall - while an inertial mass object is in cosmological acceleration.

In the principle of equivalence in 2(a) the gravitational mass of the object is in the accelerating frame of reference of gravitational freefall with respect to background spacetime. In cosmological freefall it is spacetime itself that is the accelerating frame of reference of the impressional inertial mass of an object.

An inertial mass object has been here-proposed to be material and therefore visible. It is proposed that an impressional inertial mass object is immaterial, and would therefore be invisible. Since an impressional inertial mass object possesses equivalent gravitational mass the object would of course be gravitationally detectable. It is proposed that impressional inertial mass objects are the dark matter in the universe.

Since inertial mass objects are here-proposed to be material, they can therefore collide with and scatter inertial mass objects; while since impressional inertial mass objects are proposed to be immaterial, they therefore cannot scatter other impressional inertial mass objects or inertial mass objects. Also, inertial mass objects, being material, are able to exert pressure, while immaterial impressional inertial mass objects should not be able to exert pressure.
Collisionless dark matter in colliding celestial systems [4], may be seen to imply the inability of immaterial impressional inertial mass of dark matter to scatter the material inertial mass of visible matter. Also, the acoustic oscillations peaks profile in the power spectrum of the CMBR [5], implies the pressurelessness of impressional inertial mass of dark matter. It remains to be seen if the null result in natural conditions continues to hold in ongoing and future experimental efforts to directly detect dark matter.

\section{Conclusions}

The present proposition explains the nature of dark energy, but does not make any unique prediction to prove the theory. Thus the proposition of dark energy belongs to the class of theories in physics which are in themselves unprovable rather they can only be either disproved by another theory that does make a unique prediction, or, gain acceptability due to their utilization in other works which do make unique predictions and are experimentally/observationally verified. The proposition of direct undetectability of dark matter is also in itself unprovable - it can only be disproved, if dark matter is indeed directly detected.

The present proposition adopts a classical mechanical paradigm, wherein the accelerating expansion of the universe is due to the cosmologicalization of a physical principle - that of the principle of equivalence.

In the quantum mechanical paradigm, on the other hand, the accelerating expansion of the universe is hypothesized to be due to a physical phenomenon - that of vacuum energy.

In the present classical mechanical paradigm, objects which are proposed to possess impressional inertial mass, and which are in cosmological freefall are the dark matter in the universe. While in the quantum mechanical paradigm, the most popular hypothesis considers dark matter as supersymmetric particles.

It appears that both classical and quantum mechanical paradigms are necessary for a complete theory of dark energy and dark matter.

\section{REFERENCES}

[1] A. G. Riess, A. V. Filippenko, P. Challis, A. Clocchiatti, A. Diercks, P. M. Garnavich, R. L. Gilliland, C. J. Hogan, S. Jha, R. P. Kirshner, B. Leibundgut, M. M. Phillips, D. Reiss, B. P. Schmidt, R. A. Schommer, R. C. Smith, J. Spyromilio, C. Stubbs, N. B. Suntzeff, and J. Tonry, "Observational evidence from supernovae for an accelerating universe and a cosmological constant," Astrophys. J, vol. 116, pp. 1009-1038, 1998.

[2] S. Perlmutter, G. Aldering, G. Goldhaber, R. A. Knop, P. Nugent, P. G. Castro, S. Deustua, S. Fabbro, A. Goobar, D. E. Groom, I. M. Hook, A. G. Kim, M. Y. Kim, J. C. Lee, N. J. Nunes, R. Pain, C. R. Pennypacker, and R. Quimby, "Measurements of $\Omega$ and $\Lambda$ from 42 high-redshift supernovae,” Astrophys. J, vol. 517, pp. 565-586, 1999. 
[3] C. L. Bennett, D. Larson, J. L. Weiland, N. Jarosik, G. Hinshaw, N. Odegard, K. M. Smith, R. S. Hill, B. Gold, M. Halpern, E. Komatsu, M. R. Nolta, L. Page, D. N. Spergel, E. Wollack, J. Dunkley, A. Kogut, M. Limon, S. S. Meyer, G. S. Tucker, and E. L. Wright, "Nine-year Wilkinson Microwave Anisotropy Probe (WMAP) observations: final maps and results," astro-ph/1212.5225, 2013.
[4] D. Clowe, M. Brada c , A. Gonzalez, M. Markevitch, S. Randall, C. Jones, and D. Zaritsky, "A direct empirical proof of the existence of dark matter," astro-ph/0608407v1, 2006.

[5] W. Hu, "CMB temperature and polarization anisotropy fundamentals," astro-ph/0210696v1, pp. 12, 2002. 